\title{
Engineering fidelity echoes in Bose-Hubbard Hamiltonians
}

\author{
Joshua D. Bodyfelt ${ }^{1}$, Moritz Hiller ${ }^{1,2,3}$ and Tsampikos Kottos $^{1,2}$ \\ 1 Wesleyan University, Department of Physics - 265 Church St., Middletown, CT 06459, USA \\ 2 MPI for Dynamics and Self-Organization - Bunsenstraße 10, D-37073 Göttingen, Germany \\ 3 Fakultät für Physik, Universität Göttingen - Friedrich-Hund-Platz 1, D-37077 Göttingen, Germany
}

PACS 05.30.Jp - Boson systems.

PACS 05.45.Mt - Semiclassical methods in quantum chaos.

PACS 03.65.Yz - Decoherence; open systems; quantum statistical methods.

\begin{abstract}
We analyze the fidelity decay for a system of interacting bosons described by a BoseHubbard Hamiltonian. We find echoes associated with "non-universal" structures that dominate the energy landscape of the perturbation operator. Despite their classical origin, these echoes persist deep into the quantum (perturbative) regime and can be described by an improved random matrix modeling. In the opposite limit of strong perturbations (and high enough energies), classical considerations reveal the importance of self-trapping phenomena in the echo efficiency.
\end{abstract}

While the physics of the last century was mainly characterized by great advances in understanding the properties of one-particle systems, recent experimental developments have put the effects of interacting bosons at the top of the research agenda. Among the experimental realizations are systems as diverse as micro-mechanical arrays [1], coupled Josephson Junctions [2], and Bose-Einstein Condensates (BEC) loaded in optical lattices [3], the latter being suggested as viable candidates for quantum computation.

Apart from their technological applications, these systems offer unique opportunities to experimentally investigate fundamental problems of quantum mechanics. One of the main questions is the manipulation of coherence and the stability of complex quantum dynamics under external ' (environmental) perturbations. A well-established measure for the latter is the so-called fidelity (also known as the Loschmidt Echo). It was first introduced by Peres [4] who used fidelity to study quantum-classical correspondence and identify traces of classical (chaotic or integrable) dynamics in quantized systems. Recently, fidelity has been used to quantify the corruption of quantum information [5], in the framework of quantum computation.

In this Letter, we study the fidelity decay of (ultra-) cold atoms loaded in an optical lattice (OL) or coupled microtraps subject to perturbations of the coupling: $k_{0} \rightarrow k_{0}+$ $\delta k$. In the context of OLs this perturbation is achieved by adjusting the intensity of the laser beams that create the lattice. Such systems are described by the Bose-Hubbard
Hamiltonian (BHH), which in second quantization reads

$$
\hat{H}=\frac{U}{2} \sum_{i=1}^{f} \hat{n}_{i}\left(\hat{n}_{i}-1\right)-k \sum_{\langle i, j\rangle} \hat{b}_{i}^{\dagger} \hat{b}_{j} ; \quad \hbar=1,
$$

where $f$ is the number of sites and $\langle i, j\rangle$ denotes summation over adjacent sites and $U=4 \pi \hbar^{2} a_{s} V_{\text {eff }} / m$ describes the interaction between two atoms on a single site ( $V_{\text {eff }}$ is the effective mode volume of each site, $m$ is the atomic mass, and $a_{s}$ is the $s$-wave atomic scattering length). The operators $\hat{n}_{i}=\hat{b}_{i}^{\dagger} \hat{b}_{i}$ count the number of bosons at site $i$; the annihilation and creation operators $\hat{b}_{i}$ and $\hat{b}_{i}^{\dagger}$ obey the canonical commutation relations $\left[\hat{b}_{i}, \hat{b}_{j}^{\dagger}\right]=\delta_{i, j}$. In the context of Josephson Junction arrays, $k$ is given by the Josephson energy $E_{J}$ while $U$ accounts for the Coulomb interaction of the charged bosons [6].

We will focus our presentation on the trimer $f=3$. This is the minimum BHH model that contains all the generic ingredients [7] (like classical chaotic dynamics [8]) of large BHH lattices and therefore often is used as a prototype model [9]. Our main results are summarized in fig. 1 where we plot the fidelity $F(t)$ as function of time for various perturbation strengths $\delta k$. The new striking feature is the appearance of echoes at multiples of $t_{\text {echo }}$. By analyzing the energy landscape of the perturbation operator, we are able to identify $t_{\text {echo }}$ and control the echo efficiency by an appropriate choice of the initial preparation. For moderate perturbations $\delta k$, an improved RMT modeling that incorporates the semiclassical structures of the per- 


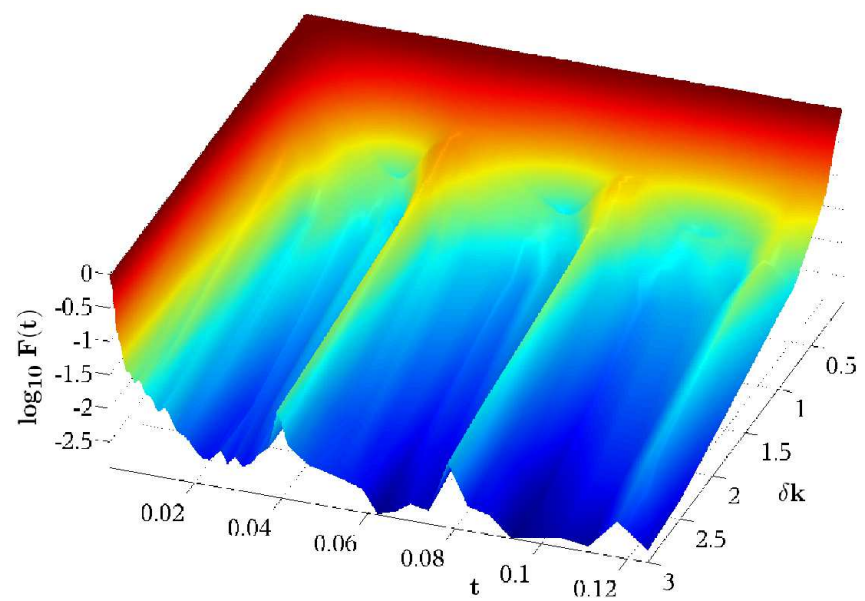

Fig. 1: (Color online) Parametric evolution of the fidelity $F(t)$ for different perturbation strengths $\delta k$ as a function of time. The fidelity exhibits echoes at multiples of $t=t_{\text {echo }}$. Here the Heisenberg time is $t_{H} \simeq 0.55$.

turbation operator can reproduce these echoes, while for larger perturbations we rely on semiclassical considerations. We show that the trajectories leading to fidelity echoes becomes more abundant at high energies, in contrast to recent experimental results on echo spectroscopy on ultra-cold atoms in atom-optics billiards [10] where it was found that the echoes do not survive in the strong perturbation limit. Our analysis indicates that this is due to self-trapping phenomena [11] and reflects a generic property of interacting bosons loaded on a lattice.

The fidelity is defined as the overlap of two states obtained after time-evolving the same initial preparation $\left|\Psi_{0}\right\rangle$ with two slightly perturbed Hamiltonians

$$
F(t)=\left|\left\langle\Psi_{0}\left|e^{i \hat{H}_{2} t} e^{-i \hat{H}_{1} t}\right| \Psi_{0}\right\rangle\right|^{2}
$$

In our analysis, we consider that $\hat{H}_{1,2}=\hat{H}_{0} \mp \delta k \hat{B}$. The unperturbed Hamiltonian $\hat{H}_{0}$ is given by eq. (1) with $k=$ $k_{0}$. In the simulations below we will use $k_{0} \approx 15, \tilde{U} \approx 280$. The number of particles in the simulations is $N=230$. The coupling operator $\hat{B}$ is defined as

$$
\hat{B}=\sum_{\langle i, j\rangle} \hat{b}_{i}^{\dagger} \hat{b}_{j} .
$$

This perturbation is similar to a momentum boost. Such a perturbation has recently been investigated [12] in the context of the fidelity, where it was found that the fidelity freezes at some finite value, as long as the boost is not too large.

Quantum mechanically, we work in the $\hat{H}_{0}$ eigenbasis. The initial preparation $\left|\Psi_{0}\right\rangle$ is chosen to be either a single eigenstate of $\hat{H}_{0}$ or a Gaussian superposition of them centered at energy $E_{0}$. A fixed assumption of this work is that the perturbation is classically small $\delta k \ll \delta k_{\mathrm{cl}}$, i.e., the corresponding classical Hamiltonians $\mathcal{H}_{0}, \mathcal{H}_{1}$ and $\mathcal{H}_{2}$ are generators of classical dynamics of the same nature.
The latter is obtained from Eq. (1) in the limit $N \gg 1$, and reads [13]

$$
\tilde{\mathcal{H}}=\mathcal{H} / N \tilde{U}=\frac{1}{2} \sum_{i=1}^{f} I_{i}^{2}-\lambda \sum_{\langle i, j\rangle} \sqrt{I_{i} I_{j}} e^{i\left(\varphi_{j}-\varphi_{i}\right)}
$$

where $\left\{I_{i}, \phi_{i}\right\}$ are the canonical action-angle variables, and $\tilde{U}=N U$. The classical dynamics is governed by the dimensionless ratio $\lambda=k / \tilde{U}[8,13]$. The other dimensionless parameter that affects the classical motion is the energy $\tilde{E}=\tilde{\mathcal{H}}$.

An important observable is the generalized force $\tilde{\mathcal{F}}(\tilde{t}) \equiv$ $-(\partial \tilde{\mathcal{H}} / \partial \lambda)=\sum_{\langle i, j\rangle} \sqrt{I_{i} I_{j}} \exp \left[i\left(\varphi_{j}-\varphi_{i}\right)\right]$ where $\tilde{t}=\tilde{U} t$ is a rescaled time. We can characterize its fluctuations by the autocorrelation function $C(\tilde{t})$. Its Fourier transform $\tilde{C}(\tilde{\omega})$ is the power spectrum of the fluctuations. Using a simple semiclassical recipe, we can connect $\tilde{C}(\tilde{\omega})$ with the band-profile of the perturbation matrix $\mathbf{B}$ [14]:

$$
\sigma^{2} \equiv\left\langle\left|\mathbf{B}_{n m}\right|^{2}\right\rangle_{E} \approx\left(N^{2} / \tilde{U}\right) \Delta \cdot \tilde{C}\left(\omega_{n m}\right) / 2 \pi
$$

where we use the basis which is determined by $\hat{H}_{0}$. Above $\langle\cdots\rangle_{E}$ indicates an averaging over an energy window $\delta E$ around some energy $E$, while $\Delta \sim \tilde{U} / N[13]$ is the mean level spacing. In cases of chaotic dynamics $\tilde{C}(\omega)$ has a cutoff frequency $\omega_{c}=2 \pi / \tau_{c}$ which is characterized by a finite correlation time $\tau_{c}$. Equation (5) implies that $\mathbf{B}$ is a banded matrix with a bandwidth $\Delta_{b}=\hbar \omega_{c}$.

Using Linear Response Theory $[15,16]$ one can obtain the following standard expression for the fidelity:

$$
F(t) \simeq 1-\delta k^{2} C(t)+\cdots \approx \exp \left(-\delta k^{2} C(t)\right),
$$

where $C(t)=\int_{0}^{t} \int_{0}^{t} C\left(t^{\prime}-t^{\prime \prime}\right) d t^{\prime} d t^{\prime \prime}$. This expression implies a short time Gaussian decay $F(t)=\exp [-8 C(0) \times$ $\left.(\delta k \cdot t)^{2}\right]$, which evolves into a long time Gaussian decay $F(t)=\exp \left[-(\sigma \delta k)^{2} t^{2}\right]$ for quantum mechanically small perturbations $\delta k \leq \delta k_{\mathrm{qm}} \sim \Delta / \sigma[16,17,20,21]$. The decay of $F(t)$ for small perturbations $\delta k \leq \delta k_{\mathrm{qm}}$, is the same irrespective of the nature (integrable or chaotic) of the underlying classical dynamics.

If the band-profile of the perturbation operator $\mathbf{B}$ were flat, we would expect $[16,17,20,21]$ an exponential decay $F(t) \sim \exp [-2 \Gamma t]$ for stronger perturbations $\delta k_{\mathrm{qm}} \leq$ $\delta k \leq \delta k_{\mathrm{prt}} \sim \tilde{U} / N$. The rate $\Gamma \sim(\sigma \delta k)^{2} / \Delta$ is given by the width of the Local Density of States (LDoS) [13]. In the perturbative regime (i.e. $\delta k \leq \delta k_{\mathrm{prt}}$ ), traditional RMT considerations $[16,17]$ can describe the fidelity decay for chaotic systems $[16,17,22]$. For even stronger perturbations $\delta k>\delta k_{\text {prt }}$, we enter the semiclassical regime $[17,20,21]$. This identification of the non-perturbative regime with the semiclassical limit can be easily understood if we realize that $\delta k_{\text {prt }} \sim \tilde{U} / N \rightarrow 0$ in the limit $N \gg 1$ (while $\tilde{U}=$ const. [13]). Then for any fixed perturbation $\delta k$, eventually $\delta k \gg \delta k_{\mathrm{prt}}$. In this regime (and provided that the classical dynamics is chaotic) the fidelity decay is exponential $F(t) \sim \exp (-\gamma t)$, with a rate given 


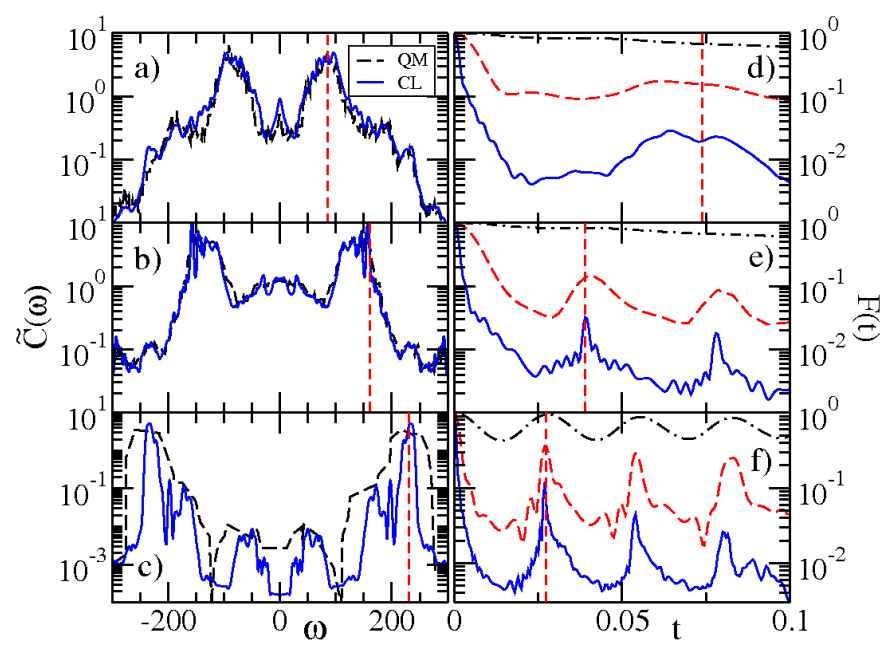

Fig. 2: (Color online) The quantum band-profile $\left\langle\left|\mathbf{B}_{n m}\right|^{2}\right\rangle \tilde{U} / N^{2} \Delta$ and classical power spectrum $\tilde{C}(\omega)$ (drawn with dashed and solid lines respectively) at various eigenvalues $\tilde{E}_{n}^{(0)}$ of the reference Hamiltonian $H_{0}$ : a) $\tilde{E}_{n}^{(0)}=0.22$, b) $\tilde{E}_{n}^{(0)}=0.26$, c) $\tilde{E}_{n}^{(0)}=0.39$. In panels d)-f) we plot the corresponding fidelity $F(t)$ for three representative values $\delta k<\delta k_{\mathrm{qm}}$ (dash-dotted line, deep perturbative regime), $\delta k_{\mathrm{qm}}<\delta k<\delta k_{\mathrm{prt}}$ (dashed line, perturbative regime), and $\delta k>\delta k_{\text {prt }}$ (solid line, non-perturbative regime). The respective numerical values are for d) and e) $\delta k=0.1,0.5,2.5$, and for f) $\delta k=0.5,2.5,7.5$. The initial preparation is an eigenstate of $H_{0}$. The vertical dashed lines in sub-figures d)-f) denote the revival time $t_{\text {echo }}$ while in a)-c) they denote the corresponding frequency $\omega^{*}=2 \pi / t_{\text {echo }}$.

by the classical Lyapunov exponent $\gamma[23]$. Our numerical data reported in figs. $2 \mathrm{t}$, e, confirm the validity of the above expectations for the BHH model (1D) in the chaotic regime.

Less clear is the situation for (predominantly) integrable systems for perturbation strengths $\delta k>\delta k_{\mathrm{prt}}$ : depending on the initial state, the fidelity decays either faster than Gaussian (when the perturbation changes the frequencies of the phase-space tori) [15], or in a power law fashion (when the primary effect of the perturbation is to change the shape of the phase-space tori) [19]. Indeed, our numerical results for the $\mathrm{BHH}$ in the (predominantly) integrable regime (high energies) confirm the above expectation (see figs. 2f, 3 and related discussion below).

Next, we focus on the appearance of fidelity echoes. In order to gain some insight we turn first to the analysis of the perturbation matrix $\mathbf{B}$ which generates the dynamics. In figs. 2 a-c we show the band-profile $\left\langle\left|\mathbf{B}_{n m}\right|^{2}\right\rangle_{E}$ (dashed line) for various energy regimes $E$. One observes that the band-profile is not flat, but exhibits pronounced structures within the bandwidth $\Delta_{b}$. A striking feature is the existence of side-bands whose position $\omega_{\text {echo }}$ increases as we increase the energy $E$. As a result $C(\tau)$ (see eq. (6) ) oscillates, leading to strong fidelity echoes at multiples of a characteristic time $t_{\text {echo }}=2 \pi / \omega_{\text {echo }}$ (see figs. $2 \mathrm{~d}-\mathrm{f}$ ). These echoes are different from the standard mesoscopic echoes at the Heisenberg time $t_{\mathrm{H}}=1 / \Delta$ of quantum systems with chaotic classical dynamics [18]. They are instead associated with "non-universal" structures that dominate the band-profile of the perturbation matrix $\mathbf{B}$ and are the fingerprints of the lattice confinement. Quantum mechanically, these are reflected in selection rules that determine to which states $\left|m\left(k_{0} \pm \delta k\right)\right\rangle$ of $H_{1,2}$ an initial state $\left|\Psi_{0}\right\rangle$ is coupled.

We consider the case where the initial preparation $\left|\Psi_{0}\right\rangle=\left|n\left(k_{0}\right)\right\rangle$ is an eigenstate of $H_{0}$ corresponding to an eigenvalue $E_{n}^{(0)}$. Here, the information about the coupling of the initial states to the perturbed states $\left|m\left(k_{0} \pm \delta k\right)\right\rangle$, is encoded in the structure of $\operatorname{LDoS} \rho_{L}(E)=$ $\sum_{m}|\langle m \mid n\rangle|^{2} \delta\left[E-\left(E_{m}-E_{n}\right)\right]$. For $\delta k \leq \delta k_{\text {prt }}$ we have that $|\langle m \mid n\rangle|^{2} \approx \delta_{n, m}+\delta k^{2}\left\langle\left|\mathbf{B}_{n m}\right|^{2}\right\rangle /\left[\Gamma^{2}+\left(E_{n}-E_{m}\right)^{2}\right]$, with a width $\Gamma \approx(\sigma \delta k)^{2} / \Delta \leq \Delta_{b}$ indicating the energy regime where most of the probability is contained [13]. For short times $t \leq \Gamma^{-1}$, this core will not have time to dephase/decay. Therefore, the fidelity will show large echo recoveries at the time the side-bands have acquired a phase of $2 \pi$, if $t_{\text {echo }} \ll \Gamma^{-1}$. The numerical data for $\delta k \leq \delta k_{\text {prt }}$ reported in figs. $2 \mathrm{~d}-\mathrm{f}$ (see dashed lines) confirm this prediction. We see that the echo efficiency (i.e. the recovery level of $F(t))$ increases as $t_{\text {echo }}$ becomes smaller [24]. Arguing along the same lines as above, we are able to explain the reduction of fidelity echoes in the non-perturbative regime, $\delta k>\delta k_{\text {prt }}$, observed in figs. 2 $\mathrm{d}-\mathrm{f}$ (see solid lines). Here, the LDoS covers the whole bandwidth, spoiling (with respect to the perturbative cases) the echo efficiency.

Similar echoes were experimentally observed in [10] for atoms in optical billiards. However, in contrast to our case (see black lines in figs. 22d-f), Ref. [10] was reporting a total loss of echoes in the semiclassical regime $\delta k \geq \delta k_{\text {prt }}$. To understand the origin of the echoes observed for the BHH model (11) for $\delta k \geq \delta k_{\text {prt }}$, we will employ semiclassical considerations. In fig. 3, we compare the quantum calculations for $\delta k>\delta k_{\text {prt }}$ with the classical fidelity

$$
F_{\mathrm{cl}}(t)=\int_{\Omega} d \mathbf{x} \rho_{-\delta k}(\mathbf{x}, t) \rho_{+\delta k}(\mathbf{x}, t)
$$

where $\rho_{ \pm \delta k}$ is the classical density function evolved under $\mathcal{H}\left(k_{0} \pm \delta k\right)$ and the integration is performed over the whole phase space $\Omega$ [25]. Although the quantum and classical calculations - as far as the echoes are concerned - agree quite well, a discrepancy on the fidelity decay between $F(t)$ and $F_{\mathrm{cl}}(t)$ for small times is also evident. In contrast to the chaotic regimes (lower energies), where for $\delta k>\delta k_{\text {prt }}$ the fidelity decay was exponential with a rate given by the classical Lyapunov exponent (see for example figs. 2d,e), here $F_{\mathrm{cl}}(t) \sim t^{-\alpha}$ while the quantum fidelity decays as $F(t) \sim t^{-3 \alpha / 2}$. The power law decay is a signature of classically (predominantly) integrable dynamics while the anomalous (faster) quantum decay is a pure quantum phenomenon, as was pointed out in [19]. According to the prediction of [17], the classical power law exponent had to be $\alpha=d$, where $d=3$ is the dimensionality of 


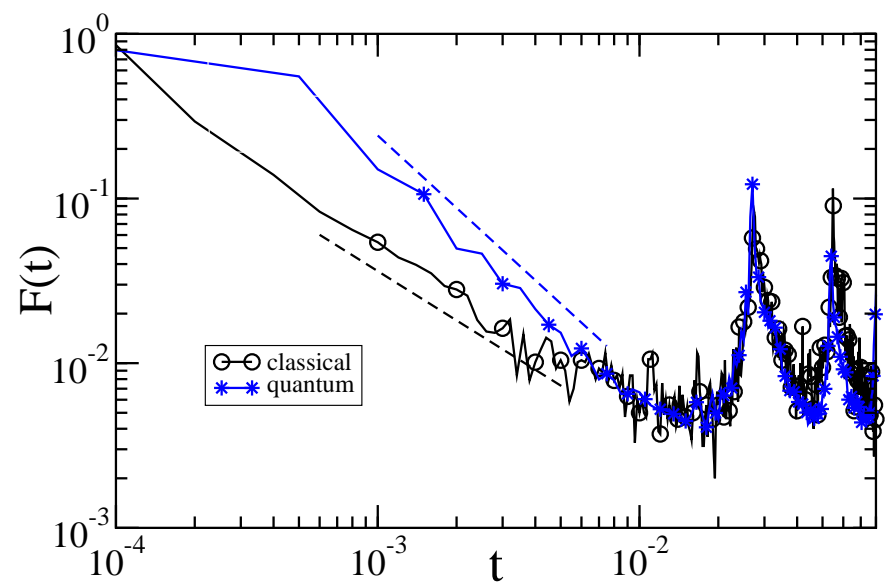

Fig. 3: (Color online) The quantum (blue line) fidelity decay $F(t)$ at $\tilde{E}_{n}^{(0)}=0.39$, plotted together with the corresponding classical (black line) fidelity decay $F_{\mathrm{cl}}(t)$, calculated from eq. (77). The perturbation strength is $\delta k=7.5>\delta k_{\text {prt }}$. The dashed lines are the best least square fits and are drawn to guide the eye. The upper one corresponds to $t^{-1.46}$ while the lower one corresponds to $t^{-0.99}$.

the system (in fact, in the case of the $\mathrm{BHH}$ model (4) we have a six-dimensional phase space with two constants of motion, namely the total number of particles $N$ and the energy $E$ and thus $d_{\text {eff }}=2$ ). However, in the high energy regime, the dynamics is dictated by self-trapping phenomena, leading to localization of particles in one site. Therefore the effective dimensionality of the system described by the Hamiltonian (44) is $d_{\text {eff }}=1$. Indeed, the best linear fit to the numerical data yields $\alpha=1$, thus confirming the above argument.

Let us now return to the analysis of the classical echoes. The classical trajectories contributing to the ensemble average of the echo, are those that - after evolving forward in time with $\mathcal{H}_{1}$ and then backwards in time with $\mathcal{H}_{2}-$ return to the vicinity of their initial position. Since $\mathcal{H}_{1}$ and $\mathcal{H}_{2}$ are different in the coupling between nearby wells, those classical trajectories that do not jump between wells will not feel the difference in the coupling terms. Therefore they retrace their forward propagation backwards in time, causing the action integral to vanish. These trapped trajectories give a perfect contribution to the echo signal. Their existence is due to both the discreteness and the nonlinearity of the underlying equations of motion. For high enough energies the configuration of bosons, localized in one lattice site persists for long times as can be seen from fig. 4. In such cases the bosons are said to be "self-trapped". As a result, the echo efficiency is increased in agreement with the numerical simulations presented in figs. 2 d-f.

We further confirm the importance of the structural average band-profile in the appearance of echoes, by evaluating the fidelity decay $F(t)$ using an improved RMT (IRMT) model [22]. While traditional RMT models involve band-profiles with matrix elements given by a Gaus-
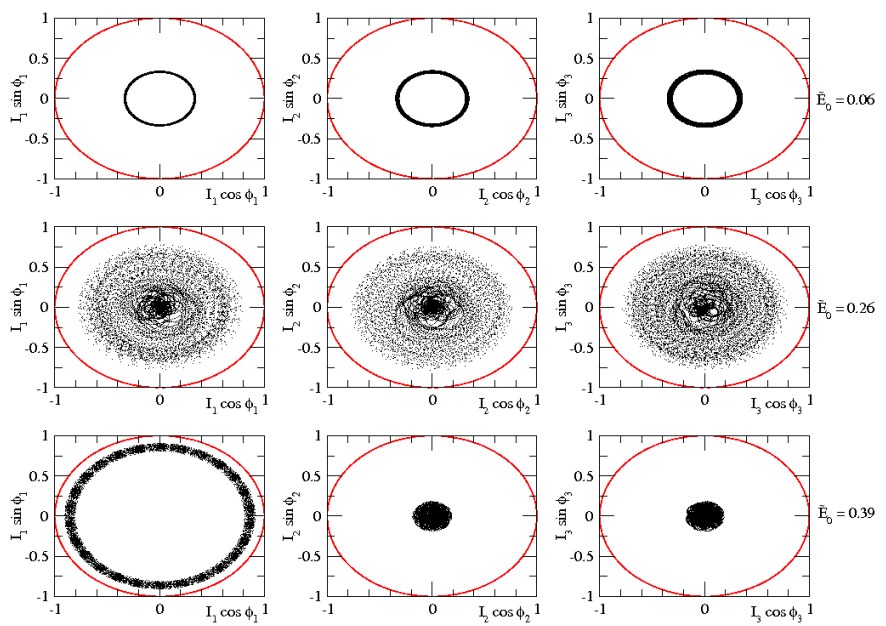

Fig. 4: (Color online) Time-evolution of $N=230$ particles (bosons) associated with various energy configurations: (top) $\tilde{E}=0.06$ close to the ground state (equipartition) of the system; (center) $\tilde{E}=0.26$ corresponding to a state in the middle of the energy spectrum and (bottom) $\tilde{E}=0.39$ associated with a high energy state. The red line indicates the accessible phase space if all particles were on the corresponding site. Note that as the energy increases, the likelihood of "trapping" particles, i.e. particles that are initially localized in one site (in the specific case $i=1$ ) and stay there for long times, increases.

sian distribution with a constant variance $\sigma^{2}$ within the bandwidth $[16,18]$, the IRMT model incorporates the band-structure associated with the dynamical system, through the semiclassical relation (5). In fig. 5 we present the results of these simulations. An excellent agreement with the fidelity calculations of the BHH (1) for the perturbative regime $\delta k<\delta k_{\mathrm{prt}}$ is evident. At the same time the IRMT modeling cannot describe the quantum results in the non-perturbative regime. Here, the echoes are related to subtler correlations of dynamical nature (self-trapping), which goes beyond the autocorrelation function $C(\tau)$ that determines the band-profile.

Finally, in fig. [5] we report our numerical calculations for the case where $\left|\Psi_{0}\right\rangle$ is a superposition of eigenstates of $H_{0}$ with a Gaussian weight around some energy $E_{0}$. We found that as the width $\sigma_{G}^{2}$ of the Gaussian preparation increases, such that the participating eigenstates of $H_{0}$ span an energy window which is wider than $\delta E$ over which the band-profile of $\mathbf{B}$ is constant (see for example Figs. 2 a-c), the echo-efficiency is suppressed. This is due to destructive interferences resulting from states located at various parts of the spectrum where the band-structure (thus the $\omega_{\text {echo }}$ ) is different. The destruction of echo efficiency becomes more pronounced in the non-perturbative

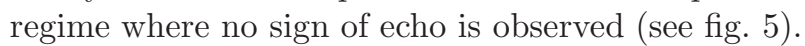

Although the above analysis is focused on the $f=3$ case, the reported results are expected to be applicable for larger lattices $f>3$ as well. Furthermore, due to the connection between fidelity decay and loss of coherence [27], our findings can be used to engineer coherence echoes 


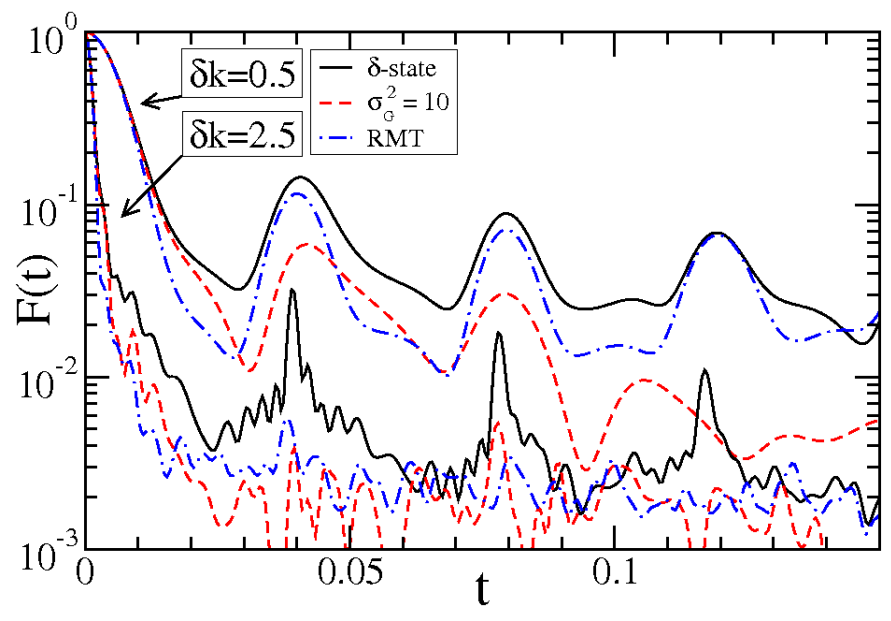

Fig. 5: (Color online) The fidelity $F(t)$ at $\tilde{E}_{n}^{(0)}=0.26$ for two perturbation strengths (upper curves $\delta k=0.5<\delta k_{\text {prt }}$, lower curves $\delta k=2.5>\delta k_{\mathrm{prt}}$ ) and various initial conditions: black solid lines correspond to $\delta$ states, dashed lines to Gaussian states with variance $\sigma^{2}=10$, and dash-dotted lines to $\delta$-states evolved under the IRMT model. We have found that within the perturbative regime, the IRMT calculations coincide with the Linear Response Theory expression from eq. (6).

of a central system coupled to a quantum bath consisting of cold atoms in an optical lattice [10,26].

We acknowledge T. Geisel for his continuous interest and support of this project. Useful discussions with D. Cohen, N. Davidson, T. Gorin, F. Izrailev, Ph. Jacquod and D. Wright are also acknowledged.

\section{REFERENCES}

[1] Sato M. et al.,Phys. Rev. Lett., 90 (2003) 44102.

[2] Trías E., Mazo J. J., and Orlando T. P.,Phys. Rev. Lett., 84 (2000) 741; Binder P. et al.,Phys. Rev. Lett., 84 (2000) 745.

[3] JaKsCh D. et al.,Phys. Rev. Lett., 81 (1998) 3108; Calarco T. et al.,Phys. Rev. A, 70 (2004) 012306; JAKSCH D. and Zoller P.,Annals of Physics, 315 (2005) 52.

[4] Peres A.,Phys. Rev. A, 34 (1984) 1610.

[5] Nielsen M. A. and ChuAng I. L.,Quantum Computation and Quantum Information (Cambridge University Press)2000.

[6] Fazio R. and van der Zant H.,Phys. Rep., 355 (2001) 235.

[7] This must be contrasted with a recent work LiU J., WANG W., Zhang C., Niu Q., and Li B.,Phys. Rev. A, 72 (2005) 063623 on a kicked (non-autonomous) dimeric BEC. Without the kicking the dynamics is integrable.

[8] Eilbeck J. C., Lomdahl P.S., and Scott A. C.,Physica D, 16 (1985) 318; E. Wright et al.,Physica D, 69 (1993) 18; Scott A. C., Lomdahl P. S., and Eilbeck J. C., Chem. Phys. Lett., 113 (1985) 29; Scotт A. C., and Eilbeck J. C.,Chem. Phys. Lett., 132 (1986) 23; ScotT A. C. BernStein L., and Eilbeck J. C.,J. Biol. Phys., 17 (1989) 1.
[9] Cucchietti F., quant-ph/0609202 (2006); DE Filippo S. Fusco Girard M., Salerno M.,Nonlinearity, 2 (1989) 477; Chefles A.,J. Phys. A, 29 (1996) 4515.

[10] Andersen M. F., Kaplan A., Grünzweig T., and Davidson N.,Phys. Rev. Lett., 97 (2006) 104102; AnderSen M. F., Kaplan A., and Davidson N.,Phys. Rev. Lett., 90 (2003) 023001; Andersen M. F., Grünzweig T., KAPlan A., and Davidson N.,Phys. Rev. A, 69 (2004) 063413.

[11] Kenkre V. M. and Tsironis G. P.,Phys. Rev. B, 35 (1987) 1473; Kenkre V. M. and Campbell D. K.,Phys. Rev. B, 34 (1986) R4959; Molina M. I. and Tsironis G. P.,Physica D, 65 (1993) 267, J. Mod. Phys. B, 9 (1995) 1899.

[12] Petitjean, C. and Bevilaqua, D.V. and Heller, E.J. and JACQOD, PH., quant-ph/0612003

[13] Hiller M., Kottos T., and Geisel T.,Phys. Rev. A, 73 (2006) 061604(R).

[14] Feingold M. and Peres A.,Phys. Rev. A, 34 (1986) 591.

[15] Prosen T. and Znidaric M.,J. Phys. A, 35 (2002) 1455; Prosen T.,Phys. Rev. E, 65 (2002) 036208.

[16] Gorin T., Prosen T., Seligman T. H., and Z̆nidaric̆ M., Phys. Rep., 435 (2006) 33; Gorin T., Prosen T., Seligman T. H.,New J. Phys., 6 (2004) 20; Prosen T., Seligman T. H., and Z̆nidaric M.,Prog.Theor.Phys.Supp., 150 (2003) 200.

[17] Jacquod Ph., Silvestrov P. G., and Beenakker C. W. J.,Phys. Rev. E, 64 (2001) 055203(R);

[18] Stöckmann H. and Schäfer R.,Phys. Rev. Lett., 94 (2005) 244101; Schäfer R., Stöckmann H. J., Gorin T., and Seligman T. H.,Phys. Rev. Lett., 95 (2005) 184102; Stöckmann H. and Schäfer R.,New J. Phys., 6 (2004) 199; Pineda C., Schäfer R., Prosen T., and Seligman T., quant-ph/0602234.

[19] Jacquod Ph., Adagdeli I., and Beenakker C. W. J.,Europhys. Lett., 61 (2003) 729.

[20] Benenti G. and Casati G.,Phys. Rev. E, 66 (2002) 066205; WAng W. and Li B.,Phys. Rev. E, 66 (2002) 056208; Wisniacki D. A. and Cohen D.,Phys. Rev. E, 2002 (046209) ; VaniceK J. and Heller E. J.,Phys. Rev. $E, 68$ (2003) 056208.

[21] Cucchietti F. M., Lewenkopf C. H., Mucciolo E. R., Pastawski H. M., and Vallejos R. O., Phys. Rev. E, 65 (2002) 046209; Jalabert R. and Pastawski H.,Phys. Rev. Lett., 86 (2001) 2490.

[22] Cohen D. and Kottos T.,Phys. Rev. E, 63 (2001) 036203; Hiller M., Cohen D., Geisel T., and Kottos T.,Annals of Physics, 321 (2006) 1025; Hiller M., Kottos T., Cohen D., and Geisel T.,Phys. Rev. Lett., 92 (2004) 010402.

[23] In fact, the perturbation-independent decay of $F(t)$ appears only for some special sets of initial states, like narrow wave packets $[17,21]$. This has to be contrasted with the perturbative regime $\delta k<\delta k_{\text {prt }}$, where the $F(t)$-decay is qualitatively the same for any initial preparation.

[24] We have checked that in the cases presented in Figs. 2]),e),f) the width $\Gamma$ remains approximately the same for corresponding $\delta k$-values.

[25] In the actual calculation the integration is done over an energy shell of width $d E$. We have checked that the numerical procedure is stable with respect to $d E$.

[26] Cohen D., and Kottos T.,Phys. Rev. E, 69 (2004) 055201(R); Gorin T. et al.,Phys. Rev. E, 70 (2004) 042105; 
Joshua D. Bodyfelt1, Moritz Hiller1,2,3 Tsampikos Kottos1,2

Rossini D. et al., quant-ph/0605051 (2006).

[27] Cucchietti F. M., Dalvit D. A. R., Paz J. P., and Zurek W. H.,Phys. Rev. Lett., 91 (2003) 210403; Gorin T., Prosen T., Seligman T. H., and Strunz W. T.,Phys. Rev. A, 70 (2004) 042105. 